\title{
UN COMENTARIO AL TEXTO CONEIXENCES DE LES MONEDES DE LOS MEMORIALES DE PERE MIQUEL CARBONELL
}

\author{
Felipe Mateu Llopis
}

Cuando en 1867 Alois Heiss publicó el tomo segundo de su Descripción General de las Monedas Hispano-cristianas des de la invasión de los árabes, incorporó a la sección de Documentos justificativos, bajo el número XIVI, un texto de los llamados Memoriales de Carbonell al que puso este título: Catálogo del tipo, ley y peso de algunas monedas que han circulado en el Principado de Cataluña. Y a pie de página añadia: *Antes de este catálogo que seguramente es de Pedro Miquel Carbonell, se halla una nota escrita de mano del mismo que, refiriéndose a una pragmática sobre monedas del Rey D. Martín de Aragôn, de 1405, dice lo siguiente: En aquest temps valia lo florí XI sols. Apres ba valgut XIII sol e buy que son en lo any MCCCCLXXXX, val XVI sol e lo ducat de Venecia XXIII sol e lo scut vell XXII sol e lo Alfonsi ducat e mig e lo real d'argent II soly.

El texto que se comenta forma parte del volumen número 35 en cuya cubierta (de un pergamino utilizado a este fin) se lee: «Memoriale privilegiorum et concessiorum factorum super moneta et super officio alcaldorum. 
La amable invitación a colaborar en Medievalia, recibida del doctor Federico Udina Martorell, director del Archivo de la Corona de Aragón, me ha hecho recordar el propósito que tenía de volver sobre el conocido texto; en 1946, tomándolo de Heiss, lo incorporé 2 mi Glosario bispánico de Numismática; pero ahora hallo la oportunidad de darlo, a la vista del original del Archivo citado, deduciendo de su estudio que el texto no es de Pere Miquel Carbonell sino anterior a él, a quien se debe parte de la nota preliminar, copiada por Heiss, sí, pero no la integridad de este importantísimo documento de la historia monetaria.

La cuestión tiene otros antecedentes. El erudito aragonés Francisco Ximénez de Urrea copió una colección de documentos y redactó diferentes estudios tocantes a un mismo tema que dejó inéditos y pasaron, con el tiempo, a conservarse en el Museo Arqueológico Nacional, de Madrid, en su Sección de Numismática y Glíptica. En 1943 lo di a conocer en Hispania, tomo III, páginas 580-597 en un artículo bajo el título «Un manuscrito referente a moneda aragonesa conservado en el Museo Arqueológico Nacional (Notas sobre la historia monetaria de Aragón)s.

En dicho artículo se dio un apartado bajo el epígrafe Una copia del Catálogo de Pere Miquel Carbonell y alli expuse que en el folio 50 de dicho manuscrito se lee: ${ }^{2}$ De las monedas del Archivo de Barcelona, por los años de 1410; y en el folio 52: «Memoria de las monedas antiguas y su valor sacadas del Archivo de Barcelona. Por Diego Monfar. Año 1636». Copióse a continuación el Catálogo atribuido a Pere Miquel Carbonell y en seguida del mismo esta nota: Predicta omnia extracta sunt et transcripta a quodam libro intitulato Memoriale privilegiorum et concessionum factarum super moneta et super officio alcaldorum recondito in Archivio Regio baj (ulie) in quodam armario sito inter duas fenestras in instancia secunda dicti Archivi signato de numero 35.

En el folio 125 del manuscrito del Museo matritense se lee: Aciavall apparen les conaxences de les monedes que val cascuna e de quina ley son. Et primo del florí de Aragó. En el margen izquierdo: De mano de Jaime García, archivero que fué en tiempo del Rey don Juan el II. Copióse a continuación, nuevamente, el Catálogo que se atribuye Pere Miquel Carbonell. Más adelante, en el folio 130 del manuscrito matritense se lee: De mano de Pere Carbonell: mancusus val dotze diners Bar. E trobas en los contractes antichs bon diu solvitur annuatim pro censu buius bospitii unud 
mancusus valens duodecim denarios monete Barcinone quemadmodum ego Carbonellus sepius comperi vidi et legi.

Por otra parte Josef Salat en su Tratado de las monedas labradas en el Principado de Cataluña (1818), tomo II, páginas 89-91, puso esta nota al citado texto que reprodujo Heiss, según se dijo al principio: Antes de este Catálogo -escribe Salat - que seguramente es de Pere Miquel Carbonell, se halla una nota escrita de mano del mismos, dice refiriéndose a una pragmática de don Martín.

En mi citado artículo de Hispania hice algunas observaciones sobre especies mencionadas en dicho Catálogo, que serán reproducidas aquí en cada moneda descrita.

Pero las parcas lo hilaron así; ni en el citado Museo pude copiar integro el referido manuscrito matritense (cuyo valor apreció don Pio Beltrán, en Obra Completa, II), la historia patria se interpuso -1936-; nj cuando, quedando lejos de aquello, hallé la tranqujlidad necesaria para volver sobre lo que vieron, cada uno por su parte, Monfar en 1636 y Salat en 1818. Sea, pues, ésta, la de ahora, la ¿última? ocasión de contemplar el tema; al doctor Udina Martorell se deberá la oportunidad de publicar nuevamente el referido Catálogo atribuido a Pere Miquel Carbonell.

Para fecharlo hay varias circunstancias:

a) La Paleografía, pues la letra no es de los años de Carbonell.

b) El mismo texto, en su lengua, que acredita ser más antiguo.

c) Ciertas lecturas que acusan no ser original sino copia; por ejemplo, leer Missa por Nissa y otras que se indican en cada caso.

d) El hecho de mencionar monedas anteriores a la acuñación del excelente, 1475, y del ducado, de 1483 y no éstos.

e) La mención que hizo Monfar, en 1636, en el folio 125: de mano de Jayme Garcia, archivero que fue en tiempo del Rey don Juan el II y la distinción que consta en el folio 130: De mano de Pere Carbonell, sobre el mancuso.

El manuscrito matritense se atribuye, por del Arco, a Francisco Ximénez de Urrea, quien en una de sus cartas, de 5 de mayo de 1638, decía prepararse para *ir a Barcelonas, pues quería ver aparte de lo mucho que hay de nuestras antigitedadess; lo trae Ricardo del Arco y Garay en La erudición aragonesa en el siglo XVII en torno a Lastanosa (1934); no debe olvidarse que el tan citado manuscrito matritense, es una miscelánea y no todo, al parecer, del mismo autor. 
Para el más fácil examen del mismo van numerados los distintos párrafos a cuya transcripción sigue el correspondiente comentario; respétase su ortografia.

1) En aquest temps valia lo flori dor $x j$ s.e apres ba valgut. Xiij s.E buy que som en l'any MCCCCLXXXXX val XVIJ s. Elo ducat de venecia XXIIIJ s.E lo scut vell .XXIJ s. El'alfonsi.j. ducat e mig E lo real d'argent .ij.s.

El original comienza propiamente tras la curva que a modo de calderón señala su título: Aci avall apparen les conaxences de les monedes que val cascuna $e$ de quina ley son. E primo del flori d'aragó.

El texto es de la misma letra que el resto del mismo, no de Carbonell, de quien hay en el folio quinto y último, la nota que se transcribe más abajo, de mano del famoso archivero, sobre el mancuso y la mazmutina.

Es un duerno de 19,5 por 14,5 centímetros de letra de tendencia cursiva, que copió un original anterior.

En lo transcrito hay dos párrafos distintos, el primero es de 1490 , como indica, puesto a manera de nota marginal, supletoria sobre la cabecera; el segundo es de un original anterior a la moneda de oro de los Reyes Católicos labrada en Sevilla en 1475, el excelente, equivalente al doble castellano, pues no lo nombra, mientras menciona el enrique, de Enrique IV de Castilla (1454-1474).

La lengua del texto está llena de atcaísmos ortográficos, vey/por vell, conaxenca por coneixenga; banj por $n$ 'bi ba; Mallorqua por Mallorques, etc.

Ciertas formas braquigráficas no fueron leídas exactamente en la transcripción dada por Heiss, como se verá en el noble.

2) Lo florí d'aragó ha aytal conaxenfa que de la una part es Sant Johan e d'altra part una flor de lir.es de ley de .xviii.quirats.pesa hrviji.grans. $e$ val si es de pes.xj.sols.

La descripción es exacta y prueba la circulación del florín cuando ya existía el pacífic.

3) Noble de nau veyl ha aytal conaxenfa que de la una part ha un noble hom lo qual sta dins una nau e te en la ma una spasa e en l-altra ma te un escut ab flos de lyrs dins l-escut e ab leons. E d-altra 
part ha una creu ab corones $e a b$ leons. $E$ deu pesar .ij. florins .c.xiij.grans. Es a ley de .xoxiij quirats e iaj. grans. Val si es de pes .xax:sol. e sise- $n$ fayl res abatse- $n$. $\ddot{z} j$. diners per gra. Añade, en rojo, noble de nay veyl.

Eduardo III de Inglaterra (1327-1377) reemplazó el llamado gold florin por el noble; para conmemorar la victoria naval sobre los franceses de Sluys, 1340, se hizo representar en un navío, como comandante de la flota que obtuvo aquélla.

Las corones y los leons que dice el texto son las lises y los leopardos, correspondientes a sus armas; la leyenda del noble es en reverso: Ihesus autem transiens per medium illorum ibat. Referencias en Schrötter, Wörterbuch der Münzkunde.

4) E mes ni ha a Anglaterra que han lo senyal del veyl pero son dits nous (palabras leídas o copiadas, como dues naus, cuando nous lleva la abreviatura de us y la sillaba no delante) e no pesen sino ij. florins .e no valen sino .xoviij, sols si son de pes.

Eduardo VI acuñó el noble en 1465; se labró también en los Países Bajos, Felipe de Flandes (1384-1404), fecha próxima a la de don Martín de Aragón, el Humano, al que se refiere la documentación mencionada en la nota de Heiss.

5) Doble cursada vella de Castella se coneix que de la una part ba un rey coronat e d-altra part ha dos leons e dos castells la un contra l'altre. E deu pesar .j.florit.e.xocij.grans. Val .Xviij. sols.es de ley de sociij quirats e iij. quarts.

Es la dobla castellana cruzada, introducida por Pedro I (13501369) y batida también por Enrique II (1369-1379). A observar la forma doble, por dobla, en todo este texto. Copió cursada por crusada.

6) Doble Castellana de la banda ha aytal conaxenga que de una part ha una barra la qual sta a travers e a cascun cap de barra ba un cap de drach. E del altre part ha dos leons $e$ dos castells, axi xom doble cursada. Deu pesar f. flori e.xoxij. grans. val. $x v$. sols .iiij. diners es a ley de xvinij quirats. 
Es la de Juan II de Castilla (1406-1454), con múltiplos de 10, 20 y 50 y divisor.

7) Doble morisque vella ha tal conaxenfa que de la una part ba letres morisques $e$ de l'altra part contrasemble. Pesa. .j. flori.xoaj.grans a ley de .xoj.quirat.val.xy.sol.viij.diners.

Para la identificación de la dobla morisque vella hay que acudir a su propia mención, la especie dobla, pues en el reinado de Fernando II de Aragón (1479-1516) y, concretamente, cuando ya se había acuñado el ducado, 1483, y el agostar siciliano, no se usaba el término morabetí para el oro circulante, sí para el impuesto o dret así llamado eran las antiguas almohades que inspiraron las granadinas.

8) Dable baladina ha aytal conaxenfa que de la una part ba letres morisques e de l'alina part sta en la dita manera e sta cadrade dins a la un cap que sta de cayre ba un senyal tal (dibuja una estrella de seis puntas) es a ley de xaj.quirat.val si es de pes .xv. sol.pega.j.flori.xoxij, grans.

Era la granadina, mencionada en el Ordenamiento de 1442; eque se labraban e habian labrado en la casa de loa moneda de Malaga e en otras partess. Véase Glosario, con referencias a Dozy, sobre el término valadies.

9) Doble fersia ha tal conaxenga que en totes parts ba letres morisques .e son aixi fetes com lo flori d'aragó .e ha'n.j de moltes leys que no's poden conexer sino tocant'les. E ban'j de.xxi.quirats $e$ mig.Valen.xv.sol.vj diners, deuen pesar.j.flori.xoiiij.grans.

Refiérese a la piedra de toque que había en todas las cecas y casas de cambistas; sobre esta práctica mi artículo \&El vocabulari medieval de l'exercici de la monederia segons documents valencianss, Butlleti de Dialectología catalana, volumen XXIV (1936), página 98-126.

Dobla fersia, tal vez por fessia o de Fes, Fas, Fez, donde se acuñaron las doblas meriníes, desde Yacub (1258-1286) a Faris (13481358) y Hafsíes Otman III (1408-1416 ?) y Abdalá (1423- ¿...?), véase Prieto Vives, sla reforma numismática de los almohadess, Miscelänea de Historia y Estudios Arabes (1915). 
En mi artículo «Les pecúnies de la Tresoreria general i el numerari corrible en temps de la guerra contra Joan IIs, en Homenatge a Antoni Rubió $i$ Lluch (E.U.C., 1936) se describen y documentan las especies alfonsí d'or; aragonesa d'argent de Sicilia; carli vell d'argent de Sicitia; cartì nou d'argent de Sicilia; diner Barcelonès; diner jaqués; diner de Valência; dobla fersia; ducat d'or bo; ducat de Cambra; ducat genovi; ducat turc; ducat venecià enric; escut florí d'aragó; florí de Sicilia; gillat de Napols; gra; òbol; pacifici; pitxol; pugesa; reyal d'argent de Barcelona; reyal d'or de Sicilia; de Juan II y Fernando II el Católico; real de València; rocabertí de Girona; sou de Barcelona; sou jaqués; sou de Rocabertins; sou de València; tari, y timbre de València.

La comparación de las monedas que entraban o salían en la Tesorería de Juan II en 1467-1479 acredita la proximidad del texto, del que fuera su original, copiado en tiempo de Jaime García, su archivero y conocido y añadido en el último folio por Pere Miquel Carbonell que lo fue de Fernando el Católico.

10) Scut vell ha aytal conaxenfa que de la una part ba un scut ab una corona gran e dins l'escut ba tres flors de lyrs. D'altra part ha una gran creu. E deu pesar.jfloriviajigrans.e si es de pes val.xv. sols. $x$.

Es el écu a la couronne o couronne d'or de Felipe VI de Francia, Valois (1328-1350), Wörterbuch. Véase Schrötter.

11) Scut nou de tholosa, ha tal conaxenga que de la una part ba un scut ab tres flors de lyrs.e de l'altra ba una gran creu sino que al costat del scut ba una corona que dien de tholosa. e son de MontpeIler e val si es de pes.xv.sol.ij. diners es a ley de .xaij.quirats e iitj.grans. e pesa un flori. E han' $j$ que tenen. ij.corones prop l'escut.

Es el de Carlos VII de Francia (1422-1461); véase Schrötter, Wörterbuch, página 115; lo que dice el texto: han'j que tenen. ij. corones prop l'escut, son los gekrönter Lilienshild zwischen 2 gekrönten Lilien.

12) Scut qui es propi de tholosa ha tal conaxenga que de la una part ba un scut ab. iij. flors de lyrs.e al costat del scut coronat ba una .o. e val si es de pes.xv. sol.vj.pesa.j.flori e vj.grans.e de l'altra part ba una gran creu axi com los altres. 
Lo cita Liciniano Sácz, Enrique IV, página 480, un escudo doro de Tholosa.

13) Scut de tornay ha aytal conaxenga que de la una part un gran escut.e de l'altra una gran crew axi com los scuts.e no's poden conexer sino en l'or.e son a ley de .xo.quirats. Pesa j.flori.iïj.grans.val. xiij.sols.Just.

El taller monetario de Tournay trabajó hasta 1489.

14) Scut de Nissa.ha tal conaxenga que son axi com los altres scuts e son quasi de la color del or del molto.e no's poden conaxer.sino en l'or. Val.xj.sols a ley de .xviij.quirats.

El moltó es el agnel, aignel, agnelet o mouton d'or de Felipe IV de Francia (1246-1314) y sucesores, que lleva en el reverso el Cordero Místico, el Agnus Dei. Véase Schrötter, página 402, con referencias a Blanchet, Engel-Serrure y ottos.

15) Ducat venecia ba tal conaxenga que de la una part es deu lo pare $a b$ esteles entorn fetes axi (dibuja una estrella) empero pus petites $e$ mils fetes e de l'altra part es sant March ab un angell agenollat. Es mester que baien la pratica de conexer l'or, car ban'j del rey qui's semblen pero han la straya a una poqua de difficultat. deu pesar .j.flori.j. gra a ley de xciij.quirats.iij.quarts val .xv. sol.viij.

En esta descripciôn que se hace del ducado veneciano se toma por ängel la figura del Dux de Venecia que se halla arrodillado ante el Evangelista.

Véase mi artículo \&El "ducado" unidad monetaria internacional oro durante el siglo XV y su aparición en la Península Ibérica. (Notas a propósito del hallazgo de Puerto de Santa María, Cádiz ), Anuario del Cuerpo Facultativo de Archiveros Bibliotecarios y Arqueólogos, volumen II (1934), página 1-34 y 2 láminas.

16) Ducat roma ba tal conaxenga que de la una part es deu lo pare ab les steles axi com lo venecia.e de l'altra part es sant March e l'algell qui sta agenollat.e al costat de la fas de deu lo pare ba una veronica. E alla bon comencen les letres diu roma caput mundi.e pesa un flori.j.gra.es a ley de .xocij.quirats.val.xv.sols. 
Como en el anterior al Dux lo considera un ângel artodillado. La Verónica es la figura en el reverso del ducado de Paulo II (14641471), en el que se lee Alma Roma rodeando la representación de Verónica ostentando la imagen del rostro del Redentor; en anverso Paulus P.P. Secundus.

17) Ducat del rey ba tal conaxenfa com to venecia si no que en les empremptes ha dificultat.e es de tal. ley e pes com lo venecia. $V$ al si es de pes. $x v$. sol.iiij.x.

Tan imprecisa descripción no facilita su identificación entre la gran variedad tipológica de la especie ducado. Al decir del rey pudo referirse al genovino en el que se lee Conradus rex romanorum, rodeando la cruz.

18) Ducat de rodes ha tal conaxenga que de la una part es sant March e de l'altra part deu lo pare e sant March te als peus una creu semblant la que porten los cavallers de Sant Johan. deu pesar .j.flori e.j.gra.val.xijf. sol.vj.diners e es a ley de.XX.quirats.

19) Ducat turch ba tal comaxenga que de una part es deu lo pare e de l'altra part es sant March axi com lo venecia .e es milt blanch d'or que diu hom cast.val.x.sol. iuij. diners.pe.j.flori.j.gta.

20) Flori de florensa se coneix que de la una part es sant Johan.e de l'altra part ha una bella flor de lyr axi com to flori d'aragó.e ban'j de grans $e$ de pochs deu pesar i.jlori.j.gra.es.a ley de .XXIIJ, quirats, itj. grans. Val.. vv. sols vj, diners.

Articulos propios referentes al florín relacionados en Bibliografia de Felipe Mateu y Llopis (Universidad de Barcelona, 1972).

21) Flori de Genova appellat genovi. ha tal conaxensa que de la una part ba una creu.e de l'altra ha un castell axi fet (dibuja toscamente uno) deu pesar.j.flori.j.g a.a ley de ,xarij.quirats val .$x v$. sols.vj.diners.

Lo llama impropiamente florí, aunque aclara que es el genovino; sobre éste véase el artículo «El ducado*, que se cita en el número 15 . 
Las especies monetarias papales en Camillo Serafini, Le Monete e le Bolle Plumbee Pontificie del Medagliere Vaticano, descritte ed illustrate (1910).

22) Flori de sena ha tal conaxenga que de una part ha una gran S.e de l'altra ha una creu axi com lo genovi.es a ley de .xxiij.quirats.ij.tergos.pesa.j.flori.j.gra.val.xv.sols.

La moneda sienesa no ofrece duda por la sigla de su topónimo; se describen en el Corpus Nummorum Italicorum. El autor del catálogo que se comenta denomina impropiamente flori de sena al ducado de la misma, que lleva en anverso la cruz quilateral y $A / p b a$ et. O.Principium et Finis y en reverso la citada letra $\mathrm{S}$ y Sena vetus civitas Virginis. Se halla en la Carte ou liste, de Anvers de 1627, comentada en Gaceta Numismática (1976), páginas 27-37. Las especies españolas de la Carte ou liste de las de oro y plata cinculantes en Amberes en 1627.

23) Flori de Cambre a tal conaxenga.que de una part es un duch qui cavalgue un cavall tot armat.e de l'altra part un helm ab un drach dins un scut.e. una serp qui es beu lo drach en j.Infant.E deu pesar.j.flori j.gra.val.xiiji.sol vj. diners a ley de .xaij.quirats.ijj.quarts.

La descripción del ducato dóro o zecchino de Francesco I sforza duque IV de Milá (1450-1466) es exacta; se ve en el C.N.I., vol. V; amplias referencias en el artículo $\star E l$ Ducados, citado arriba.

24) Flori de ri ha tal conaxenga que de la una part es sant Joban $e$ de l'altra ba un scut ab barres. Empero ban'j de moltes maneres.Jatsia tots tenguen sant Joban a la una part e de l'altra ba diverses senyals. deu pesar.j.flori.j.gra e han'j de moltes maneres de leys. les millors son de .xox.quirats.valen.xij. sol.vj.diners, han'j qui tenen lo senyal de flandes.

Bajo la denominación de flori de Ri, esto es, del Rin, el autor agrupa una serie de piezas que llevan en anverso la imagen de san Juan Bautista, posteriormente la de san Pedro, o de diferentes prelados, acuñaciones llamadas mailles de Rhyn et aultres Florins d'or forgez en Allemagne, como se diría después en la Carta de 1627; y entre ellos sólo se distinguen los que llevan el león de Flandes, que 
se acuñaban en Gante en 1434-1447; Brujas, 1454-1459 y también en Gante en 1459-1461, y 1466-1467, durante Felipe el Bueno (1419-1467).

25) Flori de bolunya. Ha tal conaxenga que de una part ba un lebo $a b$ una bandera que te als peus .e de l'altra sant Pere ab les claus en la ma. deu pesar.j.flori.j.gra.Val.xiitj.sol vj. a ley de.xocij.quirats.

Es el ducado boloñés, de Paulo II (1464-1475), descrito con exactitud: el león rampante con el vexilo y la leyenda Bononia docet. Véase «El ducado», que se citó bajo el número 15 .

26) Flori d'ongria ha tal conaxenga. que de la una part ha un rey lo qual te en la ma una ymage e de l'altra ma un polm e de l'altra part ba un scut $a b$ braf $e$ ab angels. pesa.j. flori.j.gra.val.xiiij. sol.vj.a ley de xxij.quirats.

La frase de l'altra part ha un scut ab brag e ab angels ha de entenderse que en el reverso del de Ladislaus Dei gracia rex Hungarie - Ladislao V (1453-1457) - figura un scut, escudo, con barres, fajas, heráldicas, y los llamados angels son el águila y el león de los cuarteles dos y tres, respectivamente.

En el ducado de Matías Corvino (1468-1478), rey de Bohemia, duque de Moravia, de Silesia y de Lusacia, El Santo, san Ladislao, con nimbo, cetro y globo crucífero. Véase «El ducados que se mencionó en el número 15.

El anterior artículo se completa con otro titulado *Alfonso $\mathrm{V}$ de Aragón y el Ducado de Milán», en Quaderni Ticinesi di numismática $e$ antichità classiche (1978).

27) Fflori de papa Marti fo es de Cambre ba tal conaxenfa. que de la una part ba una corona de finistres e damunt aquella ne ba una altra. e de l'altra part san P(ere) ab les claus en la ma. deu pesar.j.flori.j.gra.val.xiin.sol.vj. diners a ley de .xcij. quirats.

En el reverso la imagen de san Pedro y en el anverso el escudo del pontífice. Es el fiorino o ducato papale, de Martin V (14171431) coronado con la tiara. 
28) Fflori de papa go es de Cambre. ha tal conaxenga. que de la una part es sant Johan.e de l'altra ba una cama en mig de un scut.e deu pesar.j.flori.j.gra.val.xiizj. sol.vj. diners. a ley de .xxij.quirats.

La descripción es imprecisa; si es florín llevaria, imprescindiblemente, la flor de lis, vista por el autor en otras especies; si es ducado papal, de Cambra, la figura es san Pedro y el escudo del reverso con una ¿cama? lectura que no puede significar pierna sino tenazas o garfios con que se sujetaba la reja al arado; tal vez interpreto por esto último alguna pieza de algunas armas papales, esto es heráldicas pontificias.

29) Fflori corrent de Cambre ha tal conaxensa que de una part es sant Johan e de l'altra una flor de lyr axi com flori de florenfa. $E$ no's pot conexer sino en l'or a quina ley son los bons basten a .xxy. quirats, val.xiiij.sol.vj.pesa.j.flori.j.gra.

Eran piezas de la tipología del florín de Florencia, y de ella misma, pero con distinta ley, verificable en la piedra de toque, según indica.

30) Fflori de Pisa fo es de Cambra ha tal conaxenfa que de una part ha la Verge Maria que te son fill al bras e seu en una cadira gran e de l'altra part ha una gran aguila coronada a les ales esteses .e. deu pesar.j.flori.j, gra.val.xinij.sol.vj. diners. es a ley de xocij.grans.

Se refiere al ducado de esta ciudad a la que Federico Barbarroja parece haberle concedido ya en su tiempo (1192-1155-1196) el derecho de acuñar moneda, que labró con la imagen de Santa Maria de Pisis, ya en las piezas más antiguas y la F del emperador, sustituida luego por el águila, que se ve también en otras cecas de Italia, como Sicilia.

31) Fflori de madama ba tal conaxenfa que de la una part es sant Johan de l'altra ha una gran creu e tres petites e deu pesar.j.flori.j.gra.val.xinij.sol.vj.diners.es a ley de .xorij. quirats.

Es la moneda de la reina de Sicilia, Juana I (1343-1382). Cítalo L. Sáez, Enrique IV, tasado en 32 sueldos e inventario de Carlos III de Navarra, en 1393. En 1473-1478 equivalía a 13 sueldos 6 dineros barceloneses y a 15 sueldos. 
32) fflori de santa Helena ha tal conaxenfa que de una part ba una testa de bome e de l'altra ba un angell ab una creu en la ma. deu pesar.j.flori.j.gra.val.xvj.sol.vj. diners es a ley de .xoiij.quirats iij.quarts.

La precedente descripción, tan imprecisa, acredita que su autor era un cambista, atento a la ley y peso de las piezas y no a sus intitulaciones; una testa de home puede ser la de Ercole I de Este (14571505) y el angell ab una creu en la ma, la figura del Redentor resucitado, como se ve en los ducados de Ferrara. Véase el artículo «Alfonso V de Aragón y el Ducado de Milán», citado, página 313.

33) fflori de lucha. ha tal conaxenga. que de la una part es sant Pere qui te les claus en la ma. e de l'altra part ha un cap de rey val xiiji.sol. vj. diners. a ley de .xoitij. quirats.j.ters.

Al llamado fiorino d'oro de Lucca, que pesaba entre 3,44 y 3,54 gramos y al florín de la República de 1369, sucedió el duacaso largo o zecchino, con el Santo Vulto, en anverso, el cap de rey que dice el autor, porque aquél va coronado; acuñaciones con san Martín en reverso; en C.N.I., XI, Toscana, Zecche Minori.

34) flori de papa Alexandre ha tal conaxenga .que de una part ha un sol ab raigs $e$ de l'altra es sant Pere. deu pesar.j.flori.j.gra.val.xiiij.sol.ij.diners. a ley de.xxxij quirats.ij.tergos.

El papa Alexandre, de ser Alejandro V, (1409-1410); el VI lo fuera en 1492-1503. Hacia 1350 comenzó a acuñarse el fiorino $d^{\prime}$ oro del Senado Romano, con la figura de san Pedro en anverso entregando el vexillum al Senador genuflexo. Inocencio VI (1352. 1362) labró en Aviñón el ducato papale, de 3,44 gramos. Juan XXIII (1410-1415) lo acuñó en Bolonia.

35) fflori de boemia ha tal conaxenga que de una part ba un rey coronat e te una verga en la ma dreta e en l'altra ma te un pom ab una creu petita.e de l'altra part un gran lebo ab gran coha ab dos caps qui stan entertoligats .deu pesar.j.flori.j.gra.val.xiiij.sol.vj.diners.es a ley de .xotij. quirats. ij.tersos. 
La descripción que el autor hace del llamado también ducado de Bohemia, oflorín, es de las más exactas; se trata del que Karolus Dei gracia, con su imagen de frente, cetro (lo que el autor llama verga) y pomo del Imperio y en reverso Romanorum (Imperator) et Boemie rex, que es Carlos V de Alemania (1346-1378); en el reverso, en efecto, un león rampante, coronado, con la cola bífida entrelazada y en las puntas de éstas, sendas cabezas. Se cambiaba, en el curso comercial, en 1627, según la Carta ou liste de Amberes, de aquel año, dibujado bajo el epigrafe Ducats de Bobeme.

36) fflori de ri ba tal conaxenga que de una part es la verge Maria $a b$ lo fill en lo bras.e de l'altra part ha un prom ab una creu sta fet axi (dibuja un círculo partido por raya horizontal) val si es de pes.xij.sol.pesa.j.flori.j.gra a ley de .xa.grans. han'j de moltes maneres que en loch de la verge Maria tenen un bisbe $e$ altres figures. pero l'or e lo so diu.

La precedente descripción es una de las que más abonan la afirmación de que este llamado Catálogo de Carbonell no es suyo; su autor describió bajo el mismo título flori de ri el número 24. Ahora se trata de Ladislao V el Póstumo, archiduque de Austria, rey de Bohemia (1453-1457), a quien sucedió Matías Corvino (1458-1490); en el anverso del ducado de Ladislao, él, de pie, coronado, con manto, cetro y globo imperial, y Ladislaus rex y en anverso la imagen de Nuestra Señora y Ladislaus rex Ungarie, la figura del rey en anverso es la que el autor del catálogo llama un prom, por probom, prohombre.

En la última parte el autor reconoce haber de moltes maneres y añade que en loch de la verge Maria tenen un bisbe o altres figures. Con esta mención genérica incluye las que presentan la imagen del obispo, tal por ejemplo la del Príncipe elector de Treveria, el de Colonia, el de Mens y los que en la Carte ou Liste de 1627 se agrupan como florin d'or. Finalmente, el autor concluye que la única forma de conocer su ley es tocarlos, en la piedra de toque, y sonarlos, sobre la del mostrador del cambista; tal significan las palabras: l'or $e$ lo so diu.

37) ffranch a peu ha tal conaxenga que de una part ba un bome a peu armat $e$ de l'altra part ha una creu. deu pesar. $j$. flori.j.vj.grans.val.xv.sol.vj.diners.es a ley de .xorij, quirats. 
Es el franc d'or a pied de Carlos V de Francia (1364-1380). Referencias en Schrötter, Wörterbuch.

38) frranch a cavall hat tal conaxenga que de una part ba un hom a cavall armat.e de lialtra ha una gran creu. deu pesar.j.fori, vj.grans.val.xv.sol.vj. es a ley de xorij quirats.e ha prop la creu flors de lyrs.

La descripción cuadra con la del franc a cheval francés, de Carlos V (1364-1380).

39) ffranch a peu de franga. se coneix que de una part ha una gran creu axi xom l'altre franch, $e$ d'altra part ha un rey coronat $e$ seu en una cadira, te en la ma una spasa en l'altra ma te un scut pe. tit e dins l'escut ab flors de lyrs petites. e compre's per or de .xoiij. quirats e mig.pesa.j. flori.xocij.grans, val $x v$. sol vj. diners.

Es la descripción de la Chaise d'or francesa, de Felipe IV, de 1303; acuñada por Felipe VI en 1346 con diferente peso; eran las de éste las circulantes cuando se redactó el catálogo que se comenta. Referencias en Schrötter, Wörterbuch.

40) Reyal de franga ba tal conaxensa que de una part ba una gran rosa e d'altra part ha un rey lo qual te en la ma pertia. deu pesar.j.flori.vj.grans.val.xv. sol.es a ley de .xvij.quirats $1 / 2$.

Es la masse d'or de Felipe IV de Francia (1285-1314); Schrörter, Wörterbuch, lámina 13, número 236. Aquí se presenta una cuestión paleográfica: el manuscrito dice pertia, con abreviatura de per; Heiss escribío partia, en el Glosario quedó con...por incierto; considerando que el autor llamó verga al cetro, en número 35, cabe pensarse si no quiso decir pertia por pertja o percha, la que se usa en las embarcaciones fluviales, llamando aquî al cetro del rey percha, o pértiga, que es lo mismo.

41) reyal d'or de Mallorcha se coneix que de una part ha un rey coronat que te una spasa en la ma e en l'altra part te un pom ab una creu petite e de l'altra part ba una creu ab dues barres. deu pesar.j.flori.iij.grans.val.xij.sols es a ley de .xx.quirats. 
La descripción del real de oro de Mallorca de Sancho (13111321) y Pedro el Ceremonioso (1343-1387) es correcta; la creu que debe leerse petita, la que va sobre el pom o globo imperial. Véanse en Campaner y en Heiss las respectivas figuras.

42) ducat de Saboya es coneix que de una part es sant Miquel armat e un duch qui sta agenollat e de l'altra part ba un timbre. deu pesar.j.flori.j.gra.val .xiiij. sol.iiij. a ley de .xorii. quirats e mig.

Saboya acuñó el ducado en 1416-1439 con pesos de 3,47 a 3,51 gramos, inspirado en el ducado veneciano; en reverso el duca arrodillado ante san Mauricio, reciben de él el vexillum; en anverso el escudo de Saboya, que es el timbre mencionado. Véase el artículo *El ducado* citado arriba.

43) doble blanquilla morisca. se coneix que a totes parts ba letres morisques e son totes blanques. deu pesar.j.flori . ox. grans. val . $x$. sol.

Al observar el dictado morisques y no árabes, dado aquel a los moros hispanos. Véase el número 7 , no cita la ley, como en la doble morisque vella, número, 7 , ni en la baladina, número 8 , de donde la blanquilla. Era la granadina de baja ley; en una carta de Medina del Campo de 1481 se mencionan las adoblas zayenes que se tornaron blanquillas», Sáez, Enrique IV, 397.

44) Molto de Muntpeller se coneix que de una part ha un anyell ab una bandereta axi com lo anyell pasqual e de l'altra part ha una gran creu axi feta com la dels scuts. deu pesar mig flori.v. grans es a ley de .xviij quirats.val viij, sol viij.

Es el mouton d'or de Felipe IV de Francia (1286-1314) y sucesores; el de Juan II de Francia (1350-1364). Véase en Schrötter, Wörterbuch, lámina 15, número 238.

45) Son altres moltons que son dits de sant Andreu e ban los senyals dels damunt dits pero ba differencia en l'or.e aquells tals no's poden coneixer sino en l'or en la color e pesen mig flori .$x v$ grans.val.vj.sol.vj.diners, es a ley de xvj. quirats. 
El mouton d'or de France pesant ij. estrelins. xox as. se cita en la Carte ou Liste, de Amberes de 1627.

46) Timbre de Perpinyá se coneix que de la una part ba un rey vestit larch lo qual te en una ma una setre e en altra un pam ab una petita creu. e en l'altra part ba un escut ab un helm e sobre l'elm un cap de drach. deu pesar.j.flori.xj. grans val.xv. sol.vj diners a ley de .xoxj, quirats.

El timbre de Perpinyá lo identificó documentalmente Botet $\mathrm{i}$ Sisó, en Les monedes catalanes, vol. II, como de Juan I de Aragón.

47) Timbre de Valencia se coneix que de una part ha un escut $a b$ un helm $a b$ un cap de drach sobre l'elm. e de l'altra part ba un senyal reyal. deu pesar mig flori. xiïj.grans. val.viij. sol vj. diners. es a ley de .xx.quirats.

Sobre el timbre, F. Mateu y Llopis, aFlorines y timbres durante Alfonso V y Ausias March. (Documentos referentes a la Ceca de València: 1388-1456*, A.C.C.V. 196, páginas 179-225. Véase también el Glosario.

48) Gostara de Sicilia se coneix que de una part ba una aguila coronada ab les ales esteses. $e$ de l'altra part ba un escut ab senyal reyal. e no ha pes car compre's per or per tant pesa e segons l'or e segons to pes, e ley s'i val.

Este nombre deriva del augustalis de Federico II de Sicilia (1190-1250), moneda de oro; la acuñó también Pedro III el Grande, de Aragón, rex Aragonum et Sicilie, por su matrimonio con Constanza; referencias en Martinori, La moneda; Schrötter, Wörterbuch, y Botet i Sisó, Les monedes catalanes, II, 71. Fernando el Católico (1479-1516) acuñó oro en Sicilia, con su imagen sentada, en trono, en anverso y águila explayada en reverso, leyenda Ferdinandus Dei gracia rex Castelle Sicilie, Aragonum y Ferdinandus Dei gracia rex Sicilie, con el peso del ducado, 3,40 a 3,50.

49) Saluis (escrito así, peto en el margen, de letra de Carbonell, Saluts) se coneix que de la una part es la salutació de la verge maria $e$ deius los peus es l'escut ab tres flors de lyrs.e de altra part ha una 
gran creu ab un lebo ab flor de lyr. deu pesar.j.flori.j.gra.val.xiiii sol vj. es a ley de .xaciij. quirats.

Es la moneda de oro de Carlos de Anjou, de Nápoles (12661285) con la escena de la Anunciación; en Salamanca, en 1460 llamada salute, Sáez, Enrique IV, 281, en documentos catalanes saluts. Véase Schrötter, Wörterbuch, bajo salut, italiano, saluto.

50) Morabati d'or se coneix quant es de taula nou. que de la una part e de l'altra ha una crew ab letres gotiques. deu pesar.j.flori viij. grans, val xiïi sol $x$ diners a ley de .xorii quirats.

51) Morabati d'or de taula vell se coneix que de la una part e de l'altra ba una creu ab letres gotiques. deu pesar. j.flori.viij grans.val $x v$.sol.tiij. a ley de xxij.quirats.

Una y otra descripción, por la mención de que ha una creu $a b$ letres gotiques, refiérese, sin duda, al maravedí alfonsí, esto es, el de Alfonso VIII de Castilla, que lleva, en efecto, una cruz pero sólo en un lado y las letras ALF; al distinguir entre de taula nou y de taula vell $\mathrm{y}$ dar mayor valor al viejo que al nuevo, acredita la larga acuñación de esta especie. Un reciente estudio de la misma en mi artículo eMorabetino-Maravedis, en Studi in memoria di Federico Melis, volumen I, páginas 459-475.

52) Alfonsi d'or se coneix que de la una part ha un rey armat a cavall e te en la ma algada una spasa e entorn ha letres gotiques. $e$ de l'altra part ha una creu que sta per quart ab lo senyal de rey $d$ 'arago e ab les armes. deu pesar. j. ducat e mig. val tant com ducat e mig fo es .xociij, sol a ley de .xxiij.quirats.

En la moneda de oro llamada alfonsi d'Aragó V el Magnánimo, acuñada en Nápoles, en 1442 (C.N.I., XVIII). Véase Glosario y aLes pecuniess, en Homenatge a Rubió $i$ Lluch, E.U.C., volumen III.

53) Besant de Alexandria se coneix que de una part e d'altra ha letres morisques axi com la doble morisque empero les letres son pus formades. deu pesar. j.flori. es tal com lo flori d'aragó. es a ley de .xoij.quirats.val, xiij.sol vj (diners). 
Alejandría, al Iskandariyah, fue ceca fatimí, zangid, ayyubita, mameluca y otmanlî. La lista de los califas abasidas de Egipto comienza con al-Zahir en H.659 (A.D. 1260). Besant de bezant y éste de Byzantius termina con al-Mutawakkil III, H. 937 (A.D. 1520) -H-945 (A.D. 1538) en que pasó Egipto a Selim I Sultán Otmanlí.

Durante Jaime II de Aragón (1291-1327) se computaba el besante de Alejandría a razón de 14 dineros minus pugesa; González Hurterbise, Libro de Tesorería.

54) Raudell de Flandes se coneix que de una part ba un bome armat cavallcant qui te en la una ma una spasa en l'altra te un scut e baix als peus del cavall ha.iiii.letres. e de l'altra part es lo senyal de flanders ab flors de lyrs a guisa de creu. deu pesar. $j$. flori.iji.grans. val. $x v$ sol vj. a ley de .xcrij. quirats e mig.

Es el reyder o rider o ryder, y también rijder, o caballero de oro. El tipo que describe el autor cuadra con el de los Vieulx Rydre de Geldres, de la Carta ou Liste de 1627.

55) Quart de noble es coneix que de una part ha flors de lyrse de l'altra part ha senyal de Anglaterra ab flors. es tan gran com flori de Mallorqua. mas es pus prim. val vij. sol.

Véase el número 3 de esta lista.

56) Enrique se coneix que de una part ba un rey coronat qui seu en cadira ab spasa en la ma. e de l'altra part ba ij. castells e ij.lebons.pesa.j.flori.xariij. grans es a ley de .xocij. quirats, val en aquest temps.xocx.sols.

Es la moneda de oro de Enrique IV de Castilla (1454-1474); la descripción es exacta; corrían en Barcelona en 1465 y por 25 sueldos barceloneses y en 1466 por 30; en aquest temps, que dice el autor, es el de la guerra contra Juan II, de Aragón (1458-1479), un indicio más de que el autor no fue Carbonell. Sobre su curso en la Tesorería el artículo citado en número 9, «Les pecunies».

57) Son ne de altra manera que de una part ha un rey a cavall en la ma te spasa. e de la altra part te lo senyal del altre. es a ley de .xoij. quirats. pesa.j.flori.xociizj.grans val ara .oox.sols. 
La descripción es la de la moneda de oro de Enrique III de Castilla (1390-1406) a la que da más valor y documentación en L. Sáez, Enrique III y Barthe, Coldocs. bist. monetaria de España, 177.

58) Aguilotxa se coneix que de la und part es un rey coronat seent en una cadira e en cascun cap de la cadira ha un aguila e lo rey te en la una ma lo sceptre e en l'altra un polm e de l'altra part ha una aguila ab hes ales esteses, deu pesar.j.flori d'or e xij.grans. es a ley de .xociij. quirats val ara.xocyj.sol.

La descripción cuadra con el ducado de Fernando el Católico, de Sicilia, donde lo acuñó en 1479; pues que la intitulación dice Ferndinandus Dei gracia rex Castelle, Sicilie, Aragonum y Ferdinandus Dei gracia rex Sicilie, antes que el excelente de la granada, de 1497. El autor termina su serie con el pacifich que sigue; o sea antes de la creación del ducado valenciano de 1483 .

59) Pacifich se coneix, que de una part es mig rey coronat ab lo sceptre en la ma. e de la part altra un scut ab senyal reyal coronat. deu pesar un flori d'or a ley de .xx.quirats e val ara .xx. sol.

Es el pacífic introducido por don Pedro de Portugal (1464-1466), seguido por Renato de Anjou (1466-1472). Ver mi artículo Les pecunies, citado en número 9. Doc. de 1474 en España Sagrada; in auro pacificorum et quartorum; al decir val ara el autor está en el citado momento, del paso de Juan II a Fernando II, 1479.

Sigue a continuación el siguiente epígrafe:

60) Conaxenga de florins d'arago appellat de Navarra se coneix que de la part de la flor de lyr diu navarra ita dices. e son aximateix dits contrafets. val. viij. sols.

El autor no reconoce set el florín de Carlos II de Navarra (13491387); que los hubiera contrahechos no empece la legitimidad de aquellas acuñaciones reales. Véanse en Heiss.

61) fflori damproer enricat acostumadament son picats en manera que no's pot gens mostrar, abans se coneix en les letres que en altra. val vj.sol. 
Damproer es una lectura deficiente, al parecer; ¿por Ampuriarum, Empories?, ¿del Emperador?; la mención de que son picats indica una fundición, no acuñación; eran, pues, fundidos mediante la utilización de una materia, cuya identificación ha de traer la citada palabra. ¿Pudo referirse a alguno de los florines germánicos que llevan la común inscripción moneta nova áurea, más el nombre de la ciudad, no siempre fácil de interpretar, por sus formas gentilicias ya que el autor lo dice?; se coneix en les letres: el florin d'or de Bamberg presenta en anverso la figura de Sanctus Henricus Imperator, en esto, Enrique II el Santo (1002-1024), en un florín de Moneta aurea Georgi Episcopi Bambergensis, de 1506, posterior al manuscrito comentado; pudiendo referirse al citado emperador Enrique, la palabra enricat; si bien podría significar enriquecido, al dorarse el metal, innoble, en que se acuñara la imitación, fuera de donde fuere, pues enriquir, enriquit, es enriquecerse. La palabra citada, amproar recuerda el verbo emproar, poner de proa, hacer navegar la nau enfonsada de proa, escorar; la voz marinera emproar no tiene que ver con amprar, emprar, pedir prestado, utilizar por otro, que no es el dueño ¿d'amprar?

62) fflori d'alchimia (la lectura correcta es alchimia y no alcurnia); dice: se coneix que son picats quasi a manera de bombolles sclafades e son los demes blanchs valen.v. sol o .v. sol. vj diners.

Clara mención de otra especie fundida de la que se aprecian bombolles, burbujas.

63) fflori de foix se coneix que en la part de la fas de sant Johan sobre lo cap ba una spasa e alla hon stan los bous tenen girades les anques deves sant Johan e val.viii.sols.

Son los florines del Bearne, sin que no hubiera falsos; pero la legitimidad de las acuñaciones cierta. Un breve artículo ela introducción del florín en Aragón y Navarra (A propósito de un florín aragonés hallado en La Oliva) s resumió la cuestión en Principe de Viana en 1946 (XXV, pp. 777-782).

En el último folio de este manuscrito se lee, de letra auténtica de Pere Miquel Carbonell los párrafos numerados aquí, 64 y 65 .

64) Mancusus val dotze diners Barcelonesos e trobe's en los contractes actichs bon diu solvitur annuatim pro censu buius bospitij 
unus Mancusus valen duodecim denarios monete Barcin (one) quemadmodum ego Carbonellus sepius comperi vidi et legi.

65) Masmutina auri invenitur in Registro Capibrevij terre Marchionatus, nescio quid valet.

66) Besancium invenitur in registro $R$ (egis) Iac(obi) pri(mi) quaere quid valeat.

67) Agostat auri moneta valens tempore R(egis) Petri 2, 1280 , minus quam solidus.

En cuanto al 64 , mancusus, y su equivalencia en 12 dineros barceloneses es tardía, es decir, se refiere a los dineros de vellón labrados desde Alfonso II de Aragón, I de Barcelona (1162-1196).

Botet i Sisó en Les monedes catalanes, II, página 17, escribe: «D'un catàlec del valor, lley y pes de varies monedes d'or que corrien en l'any 1490, atribuit a Pere Miquel Carbonells, remitiendo a Salat, II, 8 y ss. y Apéndice LXV; reconoce que el texto aquí comentado se eatribuye» a Carbonell; de éste sólo son ciertos los párrafos transcritos aquí ahora.

En cuanto a equivalencias del mancusus, antes de la moneda de doblenc y de terno di en Numisma, número 19 (1956), «El "aurum Valencie" en los "Usatici Barchinone". Estudio de las cláusulas penales pecuniarias de los "Usatges" \$. Estos han atraído en los últimos años numerosas investigaciones, G.C. Miles; J. Bastardas; M. Mayer y otras que se relacionan en Symposium Numismático de Barcelona, II (1979), también J. Bastardas en su «Discurso de ingreso en la Real Academia de Buenas Letras».

A los números 65 y 66: Sobre la mazmudina en su función durante los siglos di el artículo *Morabati y mazmudina censuales. En especial en el Reino de Valencias, Cuadernos de la Albambra, 1011 (1974-1975), páginas 201-215.

Acerca de ella y el besante, véase « Sobre la política monetaria de Jaime I y las acuñaciones valencianas de 1247 y 1271s, en A.C.C.V. (1947) páginas 233-261 y 2 láminas.

Al número 67: Carbonell leyó agostat; su forma propia es agostar. Agostars de Sicilia. Véase el número 48.

Parece, pues, fechado y aclarado el tan conocido, desde Salat y Heiss, Catálogo atribuido a Pere Miquel Carbonell, texto del XIV. 
$\mathrm{XV}$, que resulta anterior al ejercicio del célebre Archivero de la Corona de Aragón.

Considérese de utilidad, ante la lista de monedas que corrian en el Principado, como consecuencia del comercio, recordar tambiến las de la guerra sostenida en aquellos años, a que se refieren las de la Tesorería Real. Un ejemplo de los asientos que se hacían en los Libres de definicions del Archivo del Reino de Valencia, es el que sigue:

- Segons que totes les dites rahons e altres eren en la dita carta Reyal de vostra comissio pus largament contengudes, de la administracio del qual dit offici vos havets ja comptat ab mi, es asaber, del dia que aquell empasas e començas a regir ho per tot lo derrer dia del mes de juny del any MCCCCIxv, segons que appar per albara testimonial que per mi vos en stat fet $\mathrm{e}$ ara havets me-n dat compte $\mathrm{e}$ raho del temps apres seguent, es a saber, del primer dia del mes de juliol del propdit any MCCCLxv ho per tot lo derrer dia del mes de desembre, apres seguent, en que fini lo dit any MCCCCLxv e comença l'any MCCCCLxvj, dins lo qual temps se encloen vj. mesos complits, romanint lo dit offici en vostre poder, lo compte de la qual administracio havets dat a mi scrit en .j. libre de paper, intitulat segons de la dita regencia de tresoreria general; e segons que en aquell es contengut munten les reebudes que vos posats haver fetes, per raho del dit offici de la dita regencia, dins lo dit temps de .vj. mesos, es a saber, axi d'en Diego de Leo, menor de dies, aiudant de cambra del Senyor Rey, com d'en Pere Bellver, de la ciutat de Leyda, procurador del Illustre don Alfonso d'Arago, fill del dit Senyor Rey, com d'en Lluis Sanchis, tresorer general del Illustrissimo princep fill del dit Senyor Rey; com de mossen Nicholau Pujades, artiaqua de la mar de la ciutat de Barchinona e d'en Guillem Pujades, conservador del Regne de Sicilia; com d'en Francesch Pallates, receptor del Senyor Rey en la ciutat de Tarragona; com de la universitat de la ciutat de Terol; com d'en Berenguer Mercader, batle general de Regne de Valencia; com d'en Luis de Sentangel, mercader de la ciutat de Valencia; com de diverses altres persones, per composicions que feren ab la cort e per certs sguarts contenguts en les dites reebudes e per altres rahons, en lo dit libre de vostre compte contengudes e declarades, comptats e enclosos en les dites reebudes cxxiij mill dexiiij solidos viij reyals de Valencia, et viij mill cccoxij solidos xj denatios jaccenses et cc. alfonsins d'or et x. mill cceclxvij ducats turchs et ccocloooxvij enrichs d'or, quals quantitats deviets tornar del compte primer de la administració del offici de la dita referencia de tresoreria general per vos a mi retuts, lo qual es de viij mesos, qui començaren lo primer dia del mes de noembre del any 
MCCCClxiiij e finiren per tot lo derrer dia del mes de juny apres seguent, del any MCCClxv, per vos a mi retut segons que totes les dites rebudes son en lo dit libre de vostre compte contengudes e escrites largament, per menut e en summa, quadraginta quinque milla ducentos septuaginta novem solidos octo denarios barcinonenses; et quinque mille quingentos quadraginta novern florins d'or d'Arago; et viginti octo mille quadrigentos duodecim solidos duodecim denarios jaccenses; et novem mille ducentos viginti quinque ducats venecians d'or; et tres mille nongentos sexaginta septem alfonsins d'or; et octuaginta quatuor mille quingentos octuaginta unum carlins vells de argent di Sicilia; et mille trecentos duodecim ducats d'or de cambra; et ducentos quatuordecim dobles fercies d'or, et centum decem novem mille centum decem carlins nous de argent de Sicilia; et triginta quatour mille ducentos sexaginta unum gillarts de Napols de argent; et quinquaginta quinque mille sexcentos decem aragonesos de argent de Sicilia; et centum viginti septem mille ducentos quatuordecim solidos octo denarios reyals de Valencia; et decem mille quadrigentos sexaginta septem ducats turchs d'or; et quadrigentos nonaginta sex enrichs d'or

E munten les dates pagues e messions oer vos fetes, per raho del dit offici dins lo dit temps de .vj. mesos, axi an Joan de Cordova, Marti de Burgos, Miquel de Maganya; com en Pere Roig, patro de balaner; com a Fe. rrando de Rebolledo, trinchant del dit Senyor Rey; com a-n Ferrando Reion, capita de la artelleria del Senyor Rey; com a altres persones qui serviren al dit Senyor Rey en la dita artelleria; com a-n Luis de Sentangel, mercader de la ciutat de Valencia; com a mossen Joan de Vilamari, capita general de les galeres del dit Senyor Rey; com a alguns altres patrons de galeres e de naus qui staven en servei del dit Senyor Rey; com a-n Pedro de Quellar sotscomptador de casa del dit Senyor, per raho de la sessio ordinaria e extraordinaria de casa del dit Senyor; com als domestichs familiars de la dita casa per llurs quitacions e vestits, com a homens d'armes e guietaris e homens de peu qui serviren en la guerra de Cathalunya al dit Senyor Rey per lur sou; com a diverses altres persones per specials manaments que-n haviets del dit Senyor; com encara en altres dates, pagues e sessions que us ha covengudes fer necessariament, per raho del dit offici, e en altra manera comptats e enclosos en les dites dates ccloxoxviij mille decelovij sólidos viij denarios obolum bancinonesos; et decxooxiij florins e mig d'or d'Arago, los quals haviets a cobrar del damunt dit compte primer que, segons dessus es dit, a mi haviets rebut de la administracio de la dita regencia de tresoreria general, segons que totes les dites dates, pagues e messions son en lo dit libre de vostre compte contengudes e scrites largament; e en summa undecim centos sexaginta sex mille quadringentos decem solidos sex denarios barcinonesos et novem mille octingentos octuaginta solidos reyals de Valencia; et duos mill septingentos octuaginta sex florins d'or d'Arago; et quindecim mill sexcentos viginti octo solidos octo denarios jaccenses. 
E aixi egualades les reebudes desus dites ab les propdites dates pagues e messions, romania que deviets tornar de florins d'or d'Arago, jaqueses, reyals de Valencia e altres monedes, ço es, que munten mes les dites reebudes que les dates, pagues e messions que posats haver fetes, ij mill dcclxiij florins d'or d'Arago; et xij mill declxxxiiij solidos iij denarios jaccenses; et cxvij mill cccxxxiiij solidos viij denarios Reyals de Valencia; et viiij mill ccxxv ducats venecians d'or; et iij mill dcccclxvij alfonsins d'or; et lxxxiiij mill dlxxxj carlins vells d'argent de Sicilia; et mcccxij ducats d'or de cambra; et ccviiij dobles fercies d'or; et cxviij mill cx carlins nous d'argent de Sicilia; et xxiiij mill celxj gillarts de Napols d'argent; el lv mill dex aragoneses d'argent de Sicilia; et x. mill cccclxvij ducats turchs d'or; et cccclxxxxvj enrichs d'or; les quals propdites quantitats; per affinament de compte, son per vos posades en rebuda en lo compte intitulat terç, per vos en poder meu rebut, de la dita administracio de la dita regencia de tresoreria generals. 